\title{
Analysis, adaptive control and circuit simulation of a novel finance system with dissaving
}

\author{
OURANIA I. TACHA, CHRISTOS K. VOLOS, IOANNIS N. STOUBOULOS and IOANNIS M. KYPRIANIDIS
}

\begin{abstract}
In this paper a novel 3-D nonlinear finance chaotic system consisting of two nonlinearities with negative saving term, which is called 'dissaving' is presented. The dynamical analysis of the proposed system confirms its complex dynamic behavior, which is studied by using wellknown simulation tools of nonlinear theory, such as the bifurcation diagram, Lyapunov exponents and phase portraits. Also, some interesting phenomena related with nonlinear theory are observed, such as route to chaos through a period doubling sequence and crisis phenomena. In addition, an interesting scheme of adaptive control of finance system's behavior is presented. Furthermore, the novel nonlinear finance system is emulated by an electronic circuit and its dynamical behavior is studied by using the electronic simulation package Cadence OrCAD in order to confirm the feasibility of the theoretical model.
\end{abstract}

Key words: chaotic finance system, dissaving, bifurcation diagram, Lyapunov exponent, phase portrait, adaptive control, nonlinear circuit.

\section{Introduction}

In the last decades there is a growing interest, from almost all fields of natural sciences, for phenomena that emerge from nonlinear systems exhibiting chaotic behavior $[1,2,3,4]$. Also, chaotic phenomena have also been observed in economics by the middle 80's. Since then, and especially in the last few years, a new scientific field named "Econophysics" aims to study the complex dynamics of real economic systems. This happens due to the fact that many nonlinear economic systems demonstrate rich dynamics and have sensitivity on initial conditions. Towards this direction, researchers are trying to explain with the tools from nonlinear science the main features of economic theory such as structural changes, irregular (erratic) micro- and macro-economic fluctuations.

In more details, as a first step in the study of an economic model, economists take into account only endogenous variables and so the economic model's behavior is simplified. However, such an economic model, which lacks external excitation, is analogous

The Authors are with Department of Physics, Aristotle University of Thessaloniki, Thessaloniki, GR-54124, Greece. E-mails: rtacha@auth.gr, volos@physics.auth.gr, stouboulos@physics.auth.gr, imkypr@auth.gr

Received 21.10.2015. 
to an autonomous electronic circuit. Then economists enrich the economic model with exogenous variables related with political events and physical disasters. For this reason, the complexity of these models makes accurate economic forecasting very difficult.

Furthermore, in many fields of economics, such as funding, stocks and social economics, the diversity and complexity are part of the internal structure of economic models that interact with external forces, economic or social, which have nonlinear factors. For this reason, nonlinear dynamics and especially chaotic systems have been introduced to the study of economic models. Examples of nonlinear systems used in economic models, are the well known van der Pol model [5, 6] and others [7, 8, 9, 10, 11, 12, 13, 14].

Even though, the chaotic systems have been studied for the first time by the French Mathematician Henri Poincaré at the end of the 19th century [15], chaos theory has begun to evolve in the second half of the 20th century $[16,17]$ after observing different physical systems. Studying these systems revealed that although their evolution rules and initial conditions were well-known, their future progress seemed to be arbitrary and unpredictable. That was a great revolution in modern physics, terminating with Laplace's ideas of casual determinism [18].

Since then, chaos has been observed in many systems, such as population growth in ecology [20], weather and climate [19] and in laboratory, in a great number of systems, such as electrical circuits [21], chemical reactions [23], lasers [22], fluid dynamics [24] and mechanical systems as well as magneto-mechanical devices [25]. So, chaos can explain various phenomena not only in nature, but also in laboratory and many chaotic dynamical systems can be used in different scientific fields.

In a simple economic model the relationship between consumption and saving is known. The sum of these two variables gives income. However, in the majority of economic systems that have been studied, the amount of savings is considered positive because consumption is below income. The basic question, which arises is what happens in these systems if saving is negative?

Motivated by the aforementioned question, a novel nonlinear finance system with a negative saving term, which is called "dissaving" in economics, is investigated. For this reason, well-known tools of nonlinear theory, such as the bifurcation diagram, Lyapunov exponent and phase portrait are used. Various phenomena concerning chaos theory, such as route to chaos through the mechanism of period doubling and crisis phenomena are observed. Also, an interesting scheme of adaptive control of system's behavior is presented. Furthermore, the proposed nonlinear finance system is emulated by an electronic circuit and its dynamical behavior is studied by using the electronic simulation package Cadence OrCAD.

This paper is organized as follows. In Section 2, the nonlinear finance system with dissaving and its fundamental dynamics are presented. The dynamical behavior of the system is studied by numerical simulations in Section 3. The results for the global chaos control of the novel finance chaotic system with unknown parameters using adaptive control method is derived in Section 4, while in Section 5 the circuit which emulates the proposed system as well as the simulation results of system's behavior, by using the Spice are presented. Finally, Section 6 includes the conclusions of this work. 


\section{The Novel Nonlinear Finance Model}

In 2001, a third-order dynamical model, describing a nonlinear finance system, was reported [10]. The model describes time variations of three state variables namely the interest rate $x$, the investment demand $y$, and the price index $z$. This nonlinear finance chaotic system is described by the following set of differential equations:

$$
\left\{\begin{array}{l}
\frac{d x}{d t}=z+(y-a) x \\
\frac{d y}{d t}=1-b y-x^{2} \\
\frac{d z}{d t}=-x-c z
\end{array}\right.
$$

Parameters $a, b$ and $c$ stand for: the saving amount, the cost per-investment ratio and the elasticity of demand of commercial markets, respectively. All three parameters possess a positive value $(a \geqslant 0, b \geqslant 0, c \geqslant 0)$.

Also, the finance system (1) has been studied by other researchers in the same or in other mathematical forms $[11,12,26]$, by extracting interesting results about its dynamical behavior.

In this work, the $x^{2}$ term, in the second equation of system (1), has been replaced with $|x|$, which is more accurate from the economic point of view, because from the economic theory is known, that the changing rate of the investment demand $y$ is in proportion with the interest rate $x$ and not in proportion with $x^{2}$. Also, as it is mentioned, the phenomenon of dissaving, which results by giving negative values to the paramater $a$ is studied. So, for this reason, in this work the sign of $a$ in the system (1) has also been changed and the following system (2) is finally used.

$$
\left\{\begin{array}{l}
\frac{d x}{d t}=z+(y+a) x \\
\frac{d y}{d t}=1-b y-|x| \\
\frac{d z}{d t}=-x-c z
\end{array}\right.
$$

The equilibrium points of the novel system (2) are obtained by solving the equations

$$
\begin{array}{r}
z+(y+a) x=0 \\
1-b y-|x|=0 \\
-x-c z=0
\end{array}
$$


Then it is proved that the system has three equilibria:

$$
\begin{aligned}
\left(x_{1}, y_{1}, z_{1}\right) & =\left(\frac{c+a b c-b}{c}, \frac{1-a c}{c},-\frac{c+a b c-b}{c^{2}}\right) \\
\left(x_{2}, y_{2}, z_{2}\right) & =\left(0, \frac{1}{b}, 0\right) \\
\left(x_{3}, y_{3}, z_{3}\right) & =\left(-\frac{c+a b c-b}{c}, \frac{1-a c}{c}, \frac{c+a b c-b}{c^{2}}\right)
\end{aligned}
$$

Also, it is easy to see that system (2) is invariant under the change of coordinates $(x, y, z) \longrightarrow(-x, y,-z)$. Thus, it follows that the novel nonlinear finance chaotic system (2) has rotation symmetry about the $y$-axis and that any non-trivial trajectory must have a twin trajectory.

Furthermore, the $y$-axis is invariant under the flow of the novel nonlinear finance system (2). The invariant motion along the $y$-axis is characterized by

$$
\dot{y}=1-b y
$$

which is stable, but not asymptotically stable.

\section{Simulation Results}

In this section, the numerical simulation results of the novel finance system, by employing a fourth order Runge-Kutta algorithm, are presented. As tools in this approach, some of the most well-known tools of nonlinear theory, such the bifurcation diagram, the phase portrait and the Lyapunov exponents have been used. In more details, the presented bifurcation diagrams of the variable $y$ versus the parameters $a, b, c$, are produced by using the section plane $x=0$ with $d x / d t>0$. Also, the system's Lyapunov exponents have been calculated by employing the algorithm of [27].

So, firstly in figures $1,2,10$ and 11 , the bifurcation diagrams of the investment demand $y$ versus the cost per-investment ratio $b$, for $a=1$, and for various values of $c$ are depicted. As the value of $c<1$ the system remains always in periodic state (Fig. 1), while for $c>1$ (Figs. 10 and 11) a more complex behavior emerges. In detail, for $c=1$ (Fig. 2) the system presents not only periodic behavior (Figs. 4-6) but also chaotic behavior (Figs. $7-9$ ) in specific regions of the parameter $b$. The system's complexity, in this case, has also been confirmed by the respective diagram of Lyapunov exponents versus the parameter $b$ of Fig. 3. Also, some interesting phenomena related with the nonlinear theory are observed, such as period-doubling route to chaos and crisis phenomenon [28]. The crisis phenomenon in the bifurcation diagram of Fig. 2 is observed for $b=0.296$, in which the chaotic attractor is destroyed suddenly and a periodic one is generated. 


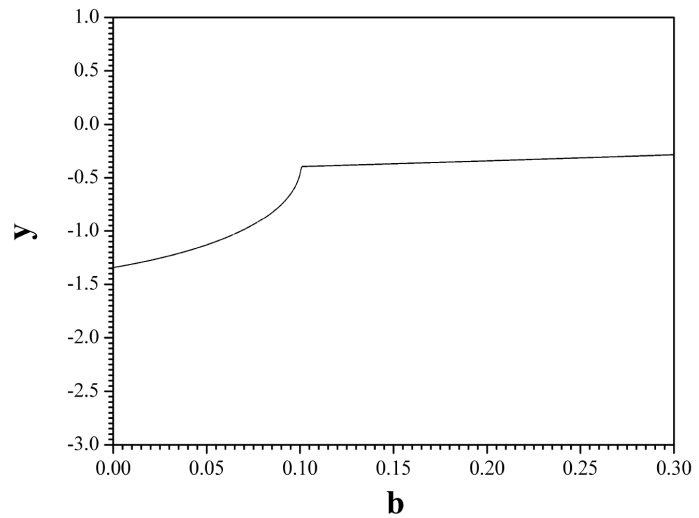

Figure 1. Bifurcation diagram of $y$ versus $b$, for $a=1, c=0.8$.

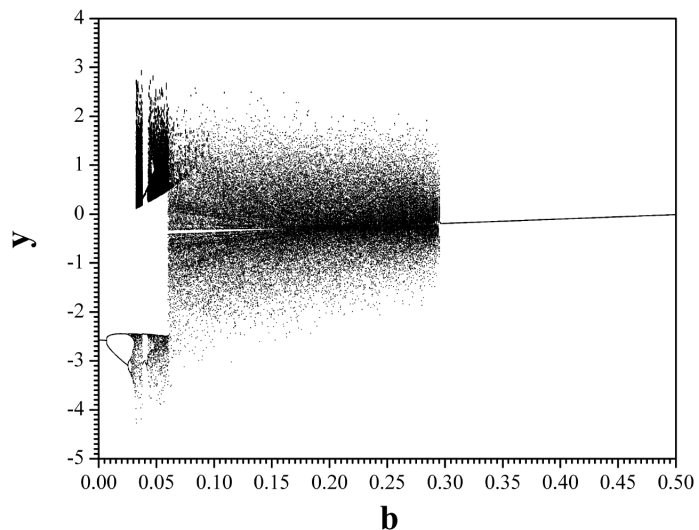

Figure 2. Bifurcation diagram of $y$ versus $b$, for $a=1, c=1$.

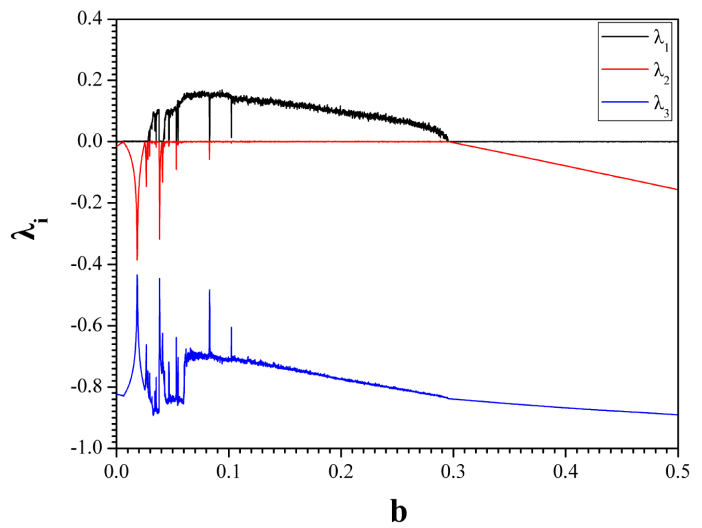

Figure 3. Diagram of Laypunov exponents versus $b$, for $a=1, c=1$. 


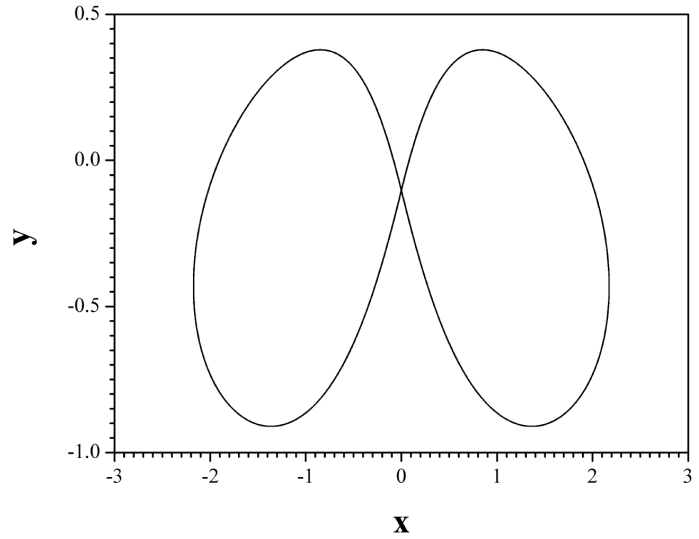

Figure 4. Phase portrait of $y$ versus $x$, for $a=1, b=0.4$ and $c=1$.

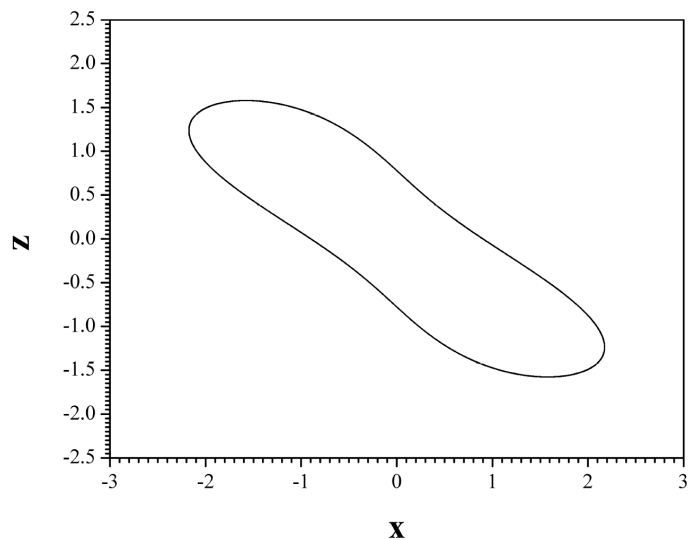

Figure 5. Phase portrait of $z$ versus $x$, for $a=1, b=0.4$ and $c=1$.

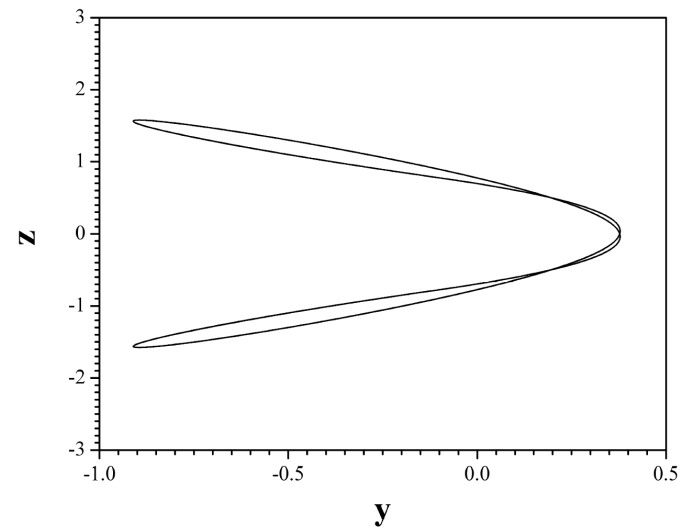

Figure 6. Phase portrait of $z$ versus $y$, for $a=1, b=0.4$ and $c=1$. 


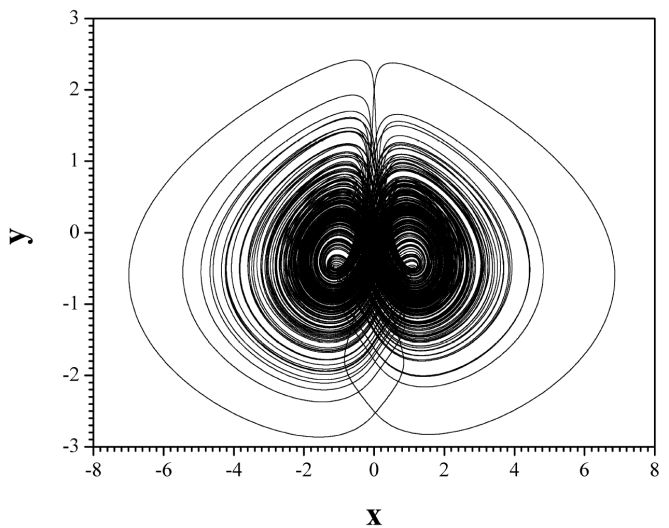

Figure 7. Phase portrait of $y$ versus $x$, for $a=1, b=0.2$ and $c=1$.

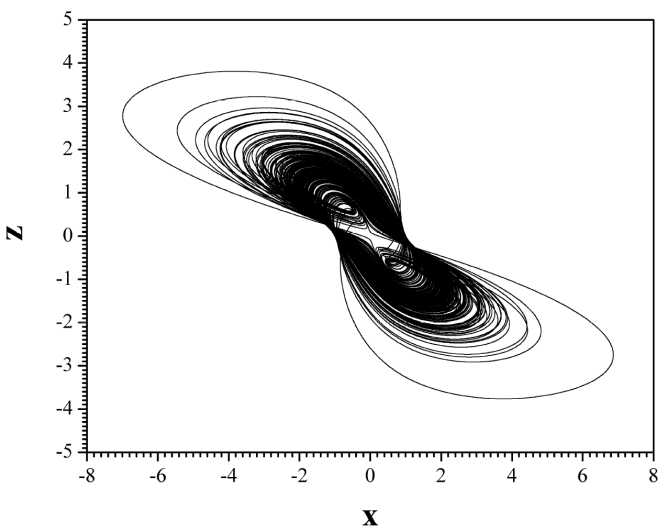

Figure 8. Phase portrait of $z$ versus $x$, for $a=1, b=0.2$ and $c=1$.

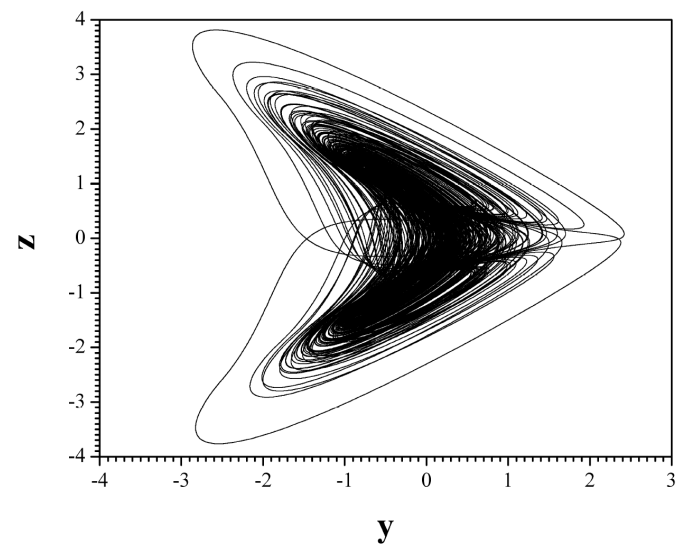

Figure 9. Phase portrait of $z$ versus $y$, for $a=1, b=0.2$ and $c=1$. 


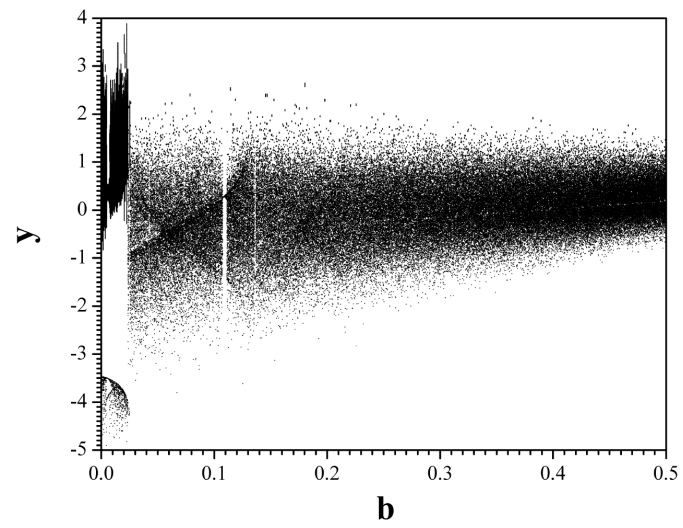

Figure 10. Bifurcation diagram of $y$ versus $b$, for $a=1, c=1.25$.

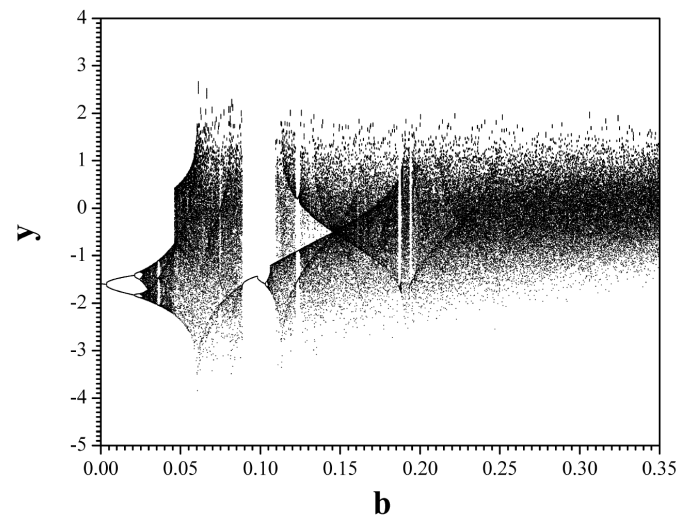

Figure 11. Bifurcation diagram of $y$ versus $b$, for $a=1, c=1.5$.

Next, the bifurcation diagrams of the investment demand $y$ versus the elasticity of demand of commercial markets $c$, for $a=1$ and for various values of $b$, have been studied (Figs. 12, 14-16). In details, for $b=0.1$ (Fig. 12) the finance system's behavior alternates between a chaotic and periodic one, as the parameter $c$ is increased. This phenomenon has been confirmed by the respective diagram of Lyapunov exponents versus the parameter $c$ (Fig. 13). However, as the value of the cost per-investment ratio $b$ increases the regions of system's periodic behavior are enlarged (Figs. 14-16).

Finally, the bifurcation diagrams of the investment demand $y$ versus the saving amount $a$, for $c=1$ and for various values of $b$, have been studied (Figs. 17, 18, 19). In details, for $b=0.1$ (Fig. 17) the finance system begins from a chaotic state and remains in this behavior, which is interrupted by small windows of periodic behavior. However, as the value of $b$ increased (i.e. for $b=0.3$ ), the region of periodic state has been enlarged and the system suddenly passes from periodic to chaotic state (Fig. 18). Finally, for values of $b>0.3$ the system remains always in a period-1 steady state (Fig. 19). 


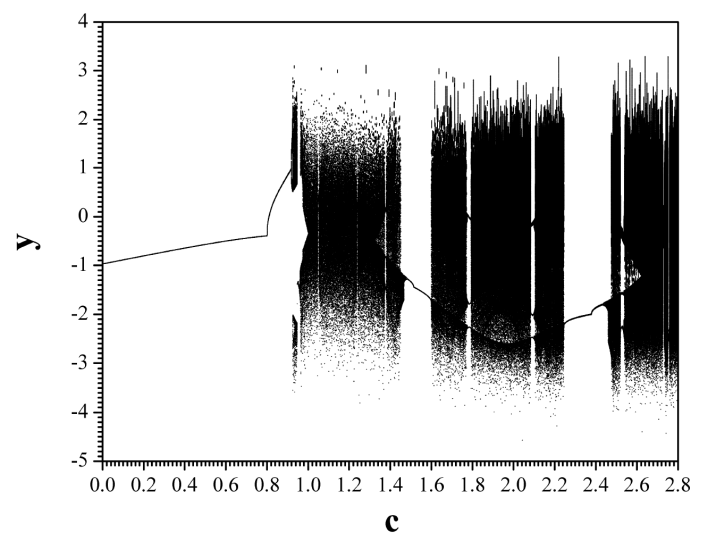

Figure 12. Bifurcation diagram of $y$ versus $c$, for $a=1, b=0.1$.

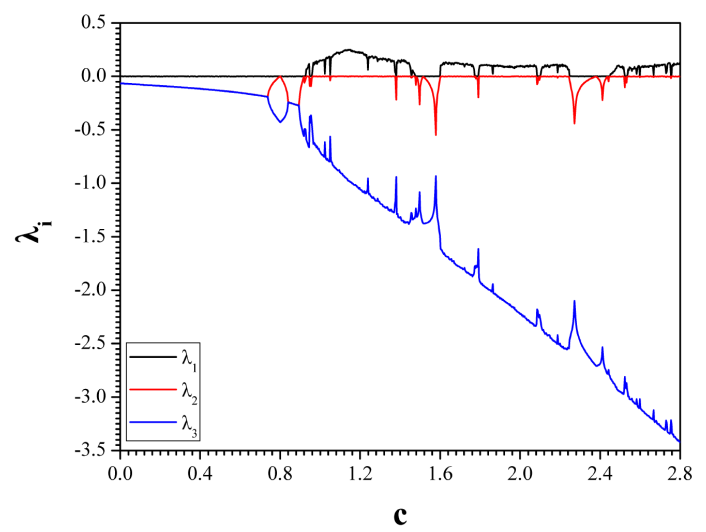

Figure 13. Diagram of Laypunov exponents versus $c$, for $a=1, b=0.1$.

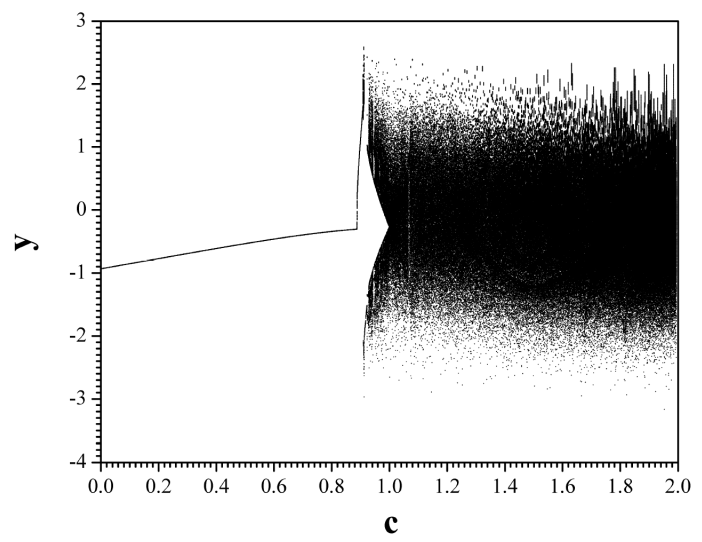

Figure 14. Bifurcation diagram of $y$ versus $c$, for $a=1, b=0.2$. 


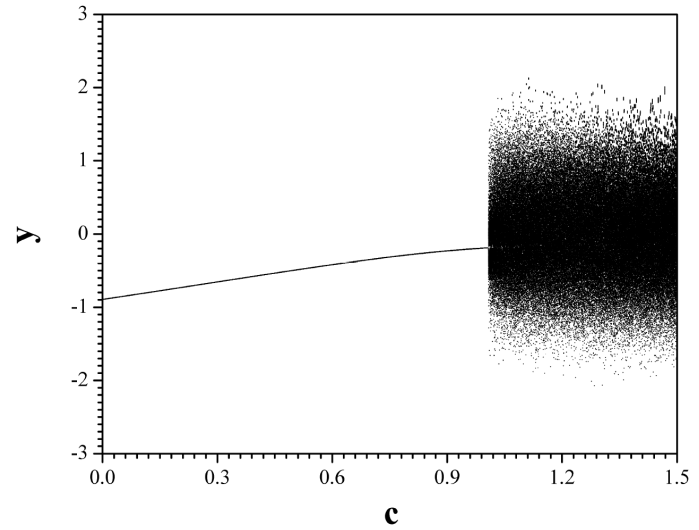

Figure 15. Bifurcation diagram of $y$ versus $c$, for $a=1, b=0.3$.

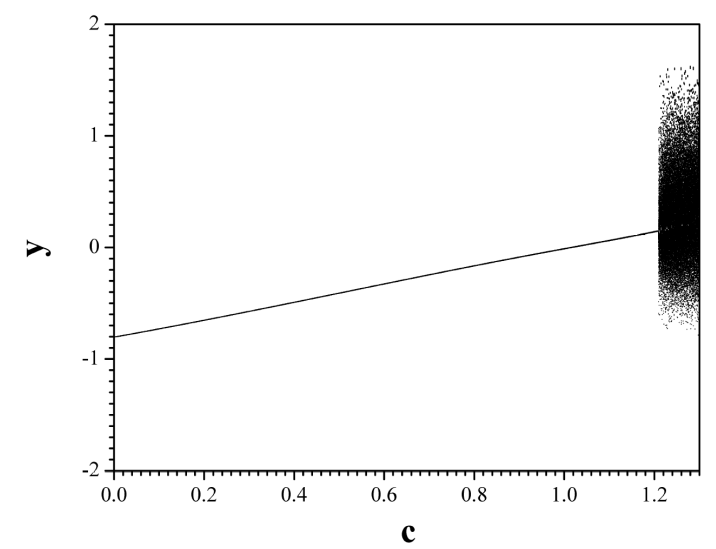

Figure 16. Bifurcation diagram of $y$ versus $c$, for $a=1, b=0.5$.

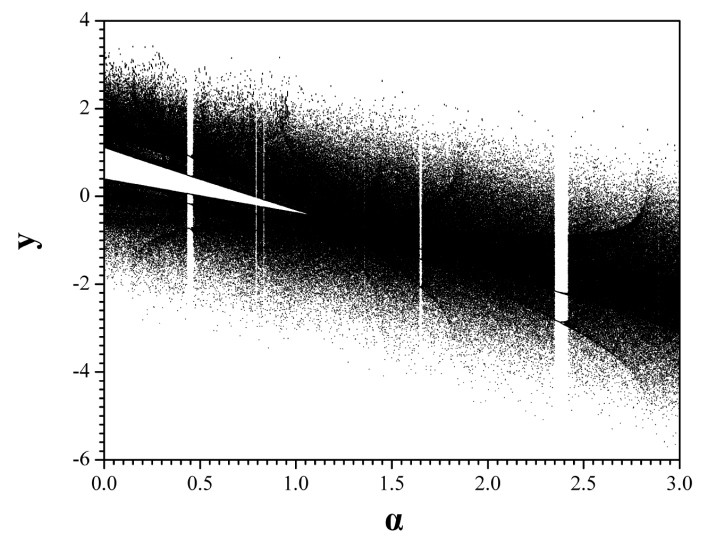

Figure 17. Bifurcation diagram of $y$ versus $a$, for $c=1, b=0.1$. 


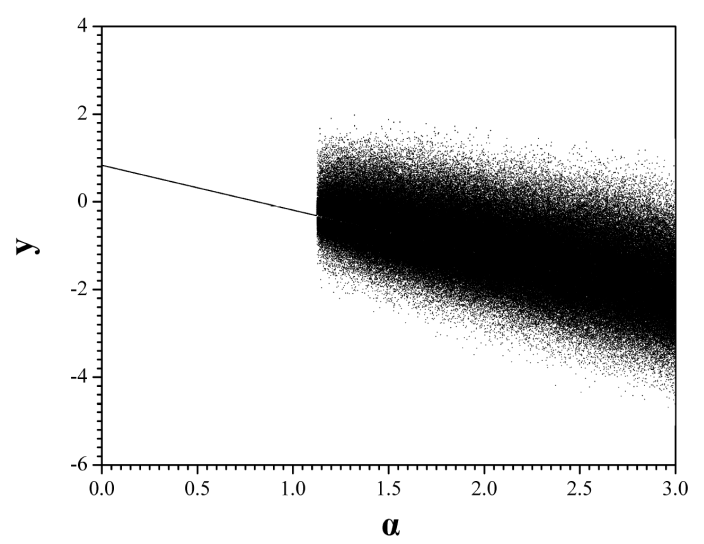

Figure 18. Bifurcation diagram of $y$ versus $a$, for $c=1, b=0.3$.

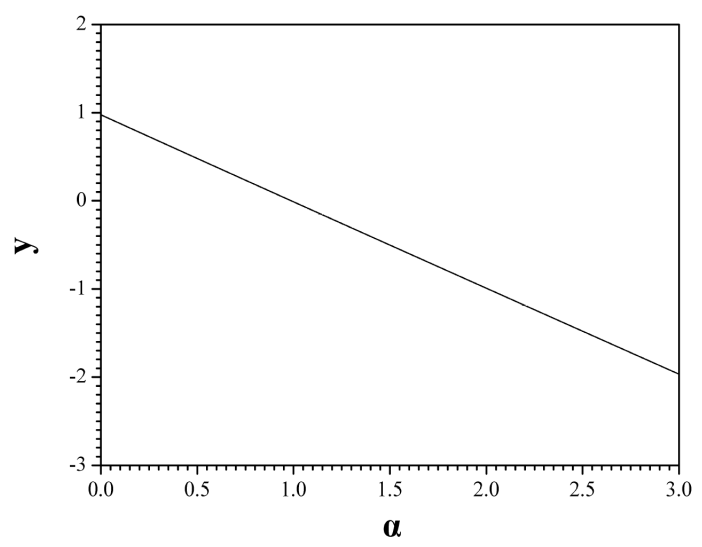

Figure 19. Bifurcation diagram of $y$ versus $a$, for $c=1, b=0.5$.

\section{Adaptive Control of the Novel Finance System}

From the results of the above simulation process, it is obvious that the inappropriate combination of the parameters in the finance system is the source that causes chaotic behavior. So, it is useful to see if the proposed finance system can be controlled into a periodic state by eliminating the unpredictable (chaotic) behavior.

For this reason, in this section, the controlled novel nonlinear finance chaotic system given by

$$
\left\{\begin{array}{l}
\frac{d x}{d t}=z+(y+a) x+U_{x} \\
\frac{d y}{d t}=1-b y-|x|+U_{y} \\
\frac{d z}{d t}=-x-c z+U_{z}
\end{array}\right.
$$


where $x, y, z$ are the states and $a, b, c$ are unknown system parameters, is studied.

The research problem of finding adaptive controls $U_{x}, U_{y}, U_{z}$ so as to regulate the states $x, y, z$ of the system (6) to desired constant values $\alpha, \beta$ and $\gamma$ respectively, is considered.

Thus, the control error is defined as:

$$
\left\{\begin{array}{l}
e_{x}(t)=x(t)-\alpha \\
e_{y}(t)=y(t)-\beta \\
e_{z}(t)=z(t)-\gamma
\end{array}\right.
$$

Then the error dynamics is determined as:

$$
\left\{\begin{aligned}
\frac{d e_{x}}{d t} & =\left(e_{z}+\gamma\right)+\left(e_{y}+\beta\right)\left(e_{x}+\alpha\right)+a\left(e_{x}+\alpha\right)+U_{x} \\
\frac{d e_{y}}{d t} & =1-b\left(e_{y}+\beta\right)-\left|e_{x}+\alpha\right|+U_{y} \\
\frac{d e_{z}}{d t} & =-\left(e_{x}+\alpha\right)-c\left(e_{z}+\gamma\right)+U_{z}
\end{aligned}\right.
$$

and the following adaptive feedback control law is considered

$$
\left\{\begin{aligned}
U_{x} & =-\left(e_{z}+\gamma\right)-\left(e_{y}+\beta\right)\left(e_{x}+\alpha\right)-\hat{a}(t)\left(e_{x}+\alpha\right)-k_{x} e_{x} \\
U_{y} & =-1+\hat{b}(t)\left(e_{y}+\beta\right)+\left|e_{x}+\alpha\right|-k_{y} e_{y} \\
U_{z} & =\left(e_{x}+\alpha\right)+\hat{c}(t)\left(e_{z}+\gamma\right)-k_{z} e_{z}
\end{aligned}\right.
$$

where $k_{x}, k_{y}, k_{z}$ are positive gain constants.

Substituting (9) into (8), the following closed-loop plant dynamics is adopted.

$$
\left\{\begin{aligned}
\frac{d e_{x}}{d t} & =[a-\hat{a}(t)]\left(e_{x}+\alpha\right)-k_{x} e_{x} \\
\frac{d e_{y}}{d t} & =-[b-\hat{b}(t)]\left(e_{y}+\beta\right)-k_{y} e_{y} \\
\frac{d e_{z}}{d t} & =-[c-\hat{c}(t)]\left(e_{z}+\gamma\right)-k_{z} e_{z}
\end{aligned}\right.
$$


The parameter estimation errors are defined as:

$$
\left\{\begin{array}{l}
e_{a}(t)=a-\hat{a}(t) \\
e_{b}(t)=b-\hat{b}(t) \\
e_{c}(t)=c-\hat{c}(t)
\end{array}\right.
$$

In view of (11), the plant dynamics (10) can be simplified as:

$$
\left\{\begin{array}{l}
\frac{d e_{x}}{d t}=e_{a}\left(e_{x}+\alpha\right)-k_{x} e_{x} \\
\frac{d e_{y}}{d t}=-e_{b}\left(e_{y}+\beta\right)-k_{y} e_{y} \\
\frac{d e_{z}}{d t}=-e_{c}\left(e_{z}+\gamma\right)-k_{z} e_{z}
\end{array}\right.
$$

Differentiating (11) with respect to $t$, the following equations are obtained.

$$
\left\{\begin{array}{l}
\frac{d e_{a}(t)}{d t}=-\frac{d \hat{a}(t)}{d t} \\
\frac{d e_{b}(t)}{d t}=-\frac{d \hat{b}(t)}{d t} \\
\frac{d e_{c}(t)}{d t}=-\frac{d \hat{c}(t)}{d t}
\end{array}\right.
$$

Next, the following quadratic candidate Lyapunov function is considered.

$$
V\left(\mathbf{e}, e_{a}, e_{b}, e_{c}\right)=\frac{1}{2}\left(e_{x}^{2}+e_{y}^{2}+e_{z}^{2}\right)+\frac{1}{2}\left(e_{a}^{2}+e_{b}^{2}+e_{c}^{2}\right)
$$

Differentiating $V$ along the trajectories of (12 and (13, the Lyapunov function (14) is written as:

$$
\begin{aligned}
& \frac{d V}{d t}=-k_{x} e_{x}^{2}-k_{y} e_{y}^{2}-k_{z} e_{z}^{2}+e_{a}\left[e_{x}\left(e_{x}+\alpha\right)-\frac{d \hat{a}}{d t}\right]+ \\
& +e_{b}\left[-e_{y}\left(e_{y}+\beta\right)-\frac{d \hat{b}}{d t}\right]++e_{c}\left[-e_{z}\left(e_{z}+\gamma\right)-\frac{d \hat{c}}{d t}\right]
\end{aligned}
$$

In view of (15), the following parameter update laws are taken as:

$$
\left\{\begin{array}{l}
\frac{d \hat{a}(t)}{d t}=e_{x}\left(e_{x}+\alpha\right) \\
\frac{d \hat{b}(t)}{d t}=-e_{y}\left(e_{y}+\beta\right) \\
\frac{d \hat{c}(t)}{d t}=-e_{z}\left(e_{z}+\gamma\right)
\end{array}\right.
$$


Next, the main result of this section will be proved.

Theorem 16 The states $x, y$ and $z$ of the novel nonlinear finance system (2) with unknown system parameters are globally and exponentially stabilized for all initial conditions to the desired constant values $\alpha, \beta$ and $\gamma$, respectivelly, by the adaptive control law (9) and the parameter update law (16), where $k_{x}, k_{y}, k_{z}$ are positive gain constants.

Proof This result will be proved by applying Lyapunov stability theory [29].

The quadratic Lyapunov function defined by (14), which is clearly a positive definite function on $\boldsymbol{R}^{6}$ is considered.

By substituting the parameter update law (16) into (15), the time derivative of $V$ is obtained as:

$$
\frac{d V}{d t}=-k_{x} e_{x}^{2}-k_{y} e_{y}^{2}-k_{z} e_{z}^{2}
$$

From (17), it is clear that $\frac{d V}{d t}$ is a negative semi-definite function on $\boldsymbol{R}^{6}$. Thus, we can conclude that the state vector $\boldsymbol{x}(t)$ and the parameter estimation error are globally bounded, i.e.

$$
\left[\begin{array}{llllll}
e_{x}(t) & e_{y}(t) & e_{z}(t) & e_{a}(t) & e_{b}(t) & e_{c}(t)
\end{array}\right]^{T} \in \mathcal{L}_{\infty}
$$

If $k=\min \left\{k_{x}, k_{y}, k_{z}\right\}$, then it follows from (17) that

$$
\frac{d V}{d t} \leqslant-k\|\boldsymbol{e}(t)\|^{2}
$$

Thus,

$$
k\|\boldsymbol{e}(t)\|^{2} \leqslant-\frac{d V}{d t}
$$

Integrating the inequality (19) from 0 to $t$, we get

$$
k \int_{0}^{t}\|\boldsymbol{e}(\tau)\|^{2} d \tau \leqslant V(0)-V(t)
$$

From (20), it follows that $\boldsymbol{e} \in \mathcal{L}_{2}$. Using (12), $\dot{\boldsymbol{e}} \in \mathcal{L}_{\infty}$ can be concluded.

Also, by using Barbalat's lemma [29], the $e(t) \rightarrow 0$ exponentially as $t \rightarrow \infty$ for all initial conditions $\boldsymbol{e}(0) \in \boldsymbol{R}^{3}$ can be concluded. Hence, it follows that the states $x, y$ and $z$ of the novel nonlinear finance system (2) with unknown system parameters $a, b$ and $c$ are globally and exponentially regulated for all initial conditions to the desired constant values $\alpha, \beta$ and $\gamma$, respectively, by the adaptive control law (9) and the parameter update law (16).

This completes the proof.

For the numerical simulation of the proposed adaptive control method the following parameter values $(a, b, c)=(0.1,0.5,1.2)$, positive gain constants $\left(k_{x}, k_{y}, k_{z}\right)=(10$, 
$10,10)$, system's initial conditions $\left(x_{0}, y_{0}, z_{0}\right)=(1,0.5,1.5)$, initial conditions of the parameter estimates $(\hat{a}, \hat{b}, \hat{c})=(0.1,0.5,1.5)$ and the desired values of the states $(\alpha, \beta$, $\gamma)=(2,1,0,5)$, are used.

In Fig. 20, the exponential convergence of the states $x, y, z$ of the novel nonlinear finance system (2) is depicted.

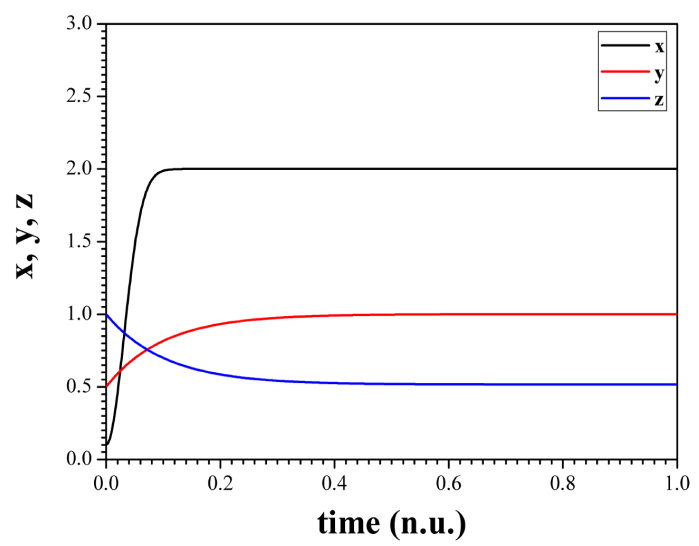

Figure 20. Time-history of the controlled states $x(t), y(t), z(t)$.

\section{Circuit Realization of the Finance System}

Circuital design of chaotic systems plays a crucial role on the field of nonlinear science not only for providing a simple experimental confirmation of phenomena related with nonlinear dynamics but also due to its applications in many engineering approaches, such as secure communication, signal processing, random bit generator, or path planning for autonomous mobile robot etc. [30, 31, 32, 33, 34, 35]. In addition, circuital implementation of chaotic systems also provides an effective approach for investigating new dynamics of such theoretical models [36, 37]. For example, time-series of chaotic signals or chaotic attractors can be observed at the oscilloscope easily or experimental bifurcation diagram can be obtained by varying the value of variable resistors $[38,39]$.

In this work, an electronic circuit (Fig. 21) is introduced for implementing system (2), which is designed by using the operational amplifiers approach [39]. It has three integrators (U1, U2 and U3) and two inverting amplifiers (U4 and U5), which are implemented with the TL084, as well as one signal multiplier (U6) by using the AD633. By applying Kirchhoff's circuit laws, the corresponding circuital equations of designed 

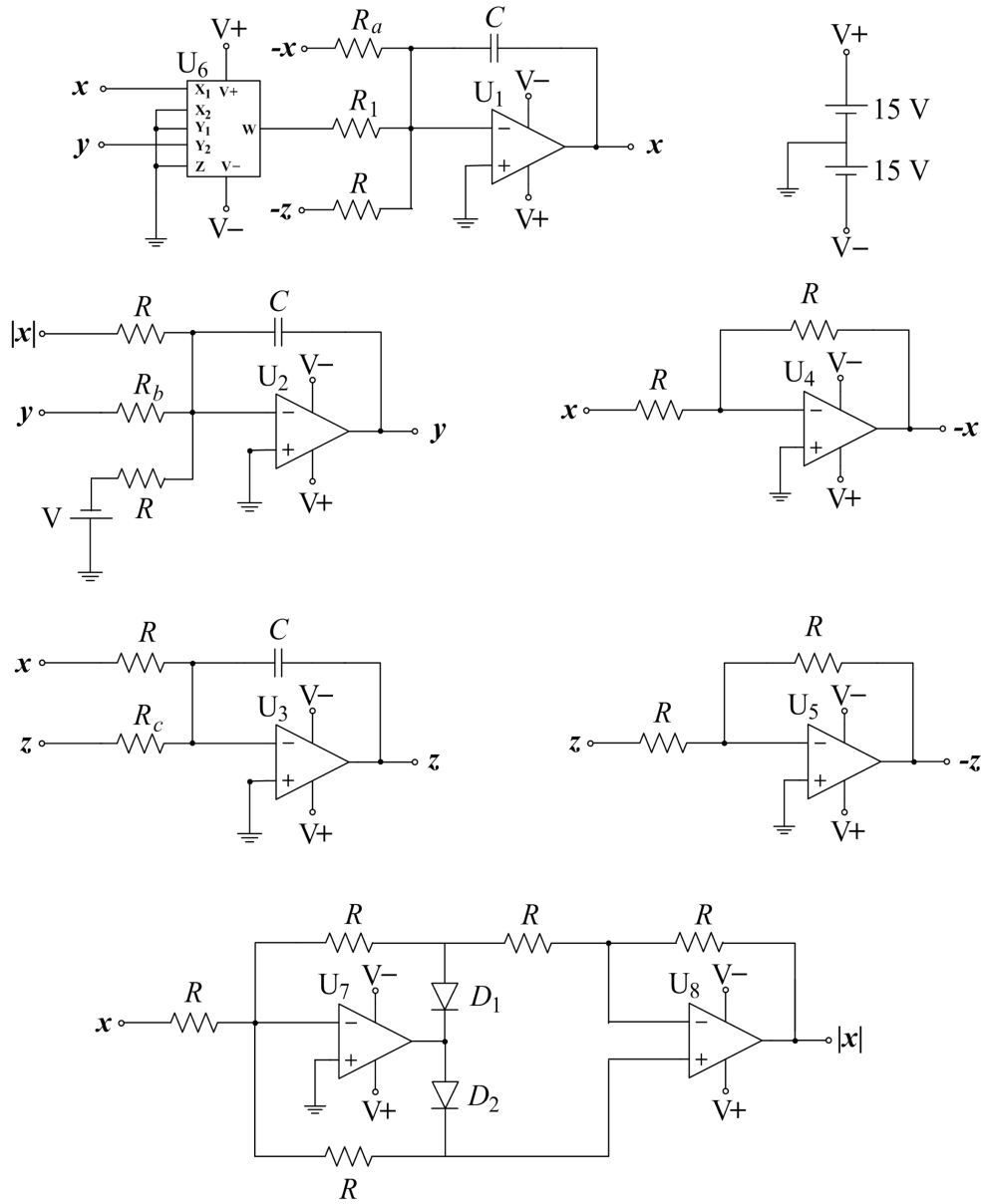

Figure 21. The schematic of the circuit that emulates the proposed finance system (2).

circuit can be written as:

$$
\left\{\begin{aligned}
\frac{d x}{d t} & =\frac{1}{R C} z+\frac{1}{R_{1} C 10 V} x y+\frac{1}{R_{a} C} x \\
\frac{d y}{d t} & =\frac{1}{R C} V-\frac{1}{R_{b} C} y-\frac{1}{R C}|x| \\
\frac{d z}{d t} & =-\frac{1}{R C} x-\frac{1}{R_{c} C} z
\end{aligned}\right.
$$

where $x, y, z$ are the voltages in the outputs of the operational amplifiers $\mathrm{U} 1, \mathrm{U} 2$ and U3. Also, the circuitry constituted by operational amplifiers U7 and U8 produces the signal $|x|$. Normalizing the differential equations of system (21) by using $\tau=t / R C$ we can see that this system is equivalent to the proposed dynamical system (2). The circuit components have been selected as: $R=10 \mathrm{k} \Omega, R_{1}=1 \mathrm{k} \Omega, C=10 \mathrm{nF}, V=1 \mathrm{~V}, D_{1}$ and $D_{2}$ 
are diodes of type $1 \mathrm{~N} 4148$ and $R_{a}, R_{b}, R_{c}$ are variable resistors, while the power supplies of all active devices are $\pm 15 V_{D C}$.

The designed circuit is implemented in the electronic simulation package Cadence OrCAD and the obtained results are displayed in Figs. 22 and 23. In more details, these figures depict the simulation phase portraits produced by the OrCAD, for $(a, b, c)=$ $(1,0.4,1)$ (Fig.22) and $(a, b, c)=(1,0.2,1)$ (Fig.23) in $x$-z plane. The comparison of the attractors, produced by OrCAD, with the respective ones, produced by the system's numerical integration (Figs. 4 and 7), proves that the theoretical attractors are similar with the circuital ones. So, the designed circuit emulates very well the proposed system's dynamic behavior.

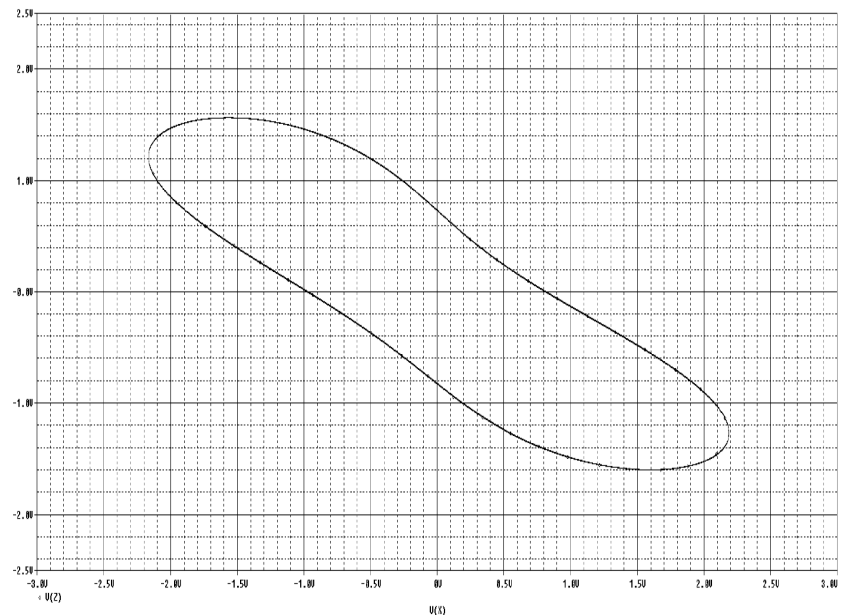

Figure 22. Phase portrait, produced in $\operatorname{OrCAD}$, of $z$ versus $x$, for $a=1, b=0.4$ and $c=1$.

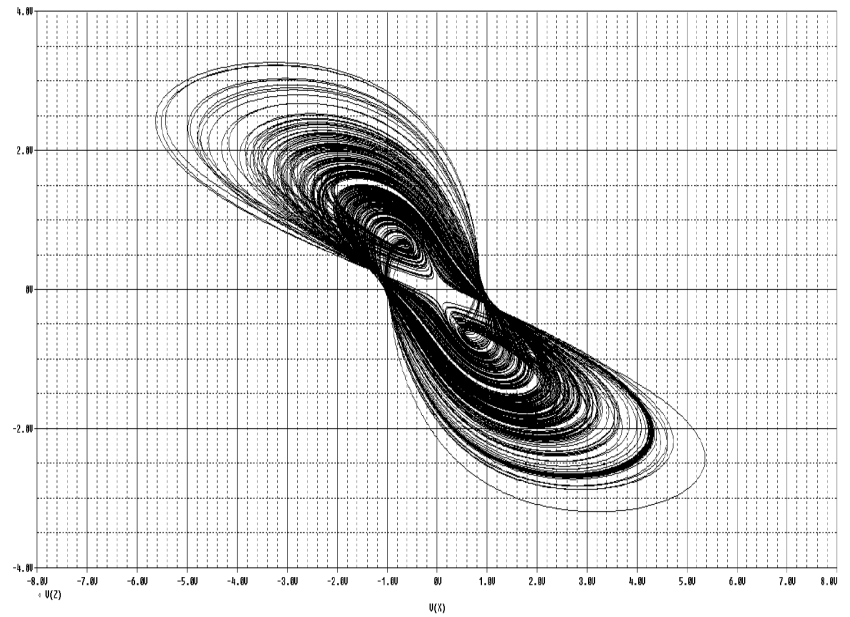

Figure 23. Phase portrait, produced in $\operatorname{OrCAD}$, of $z$ versus $x$, for $a=1, b=0.2$ and $c=1$. 


\section{Conclusions}

In the last decades, nonlinear researchers have applied nonlinear dynamic theory to economic and finance systems. It is well stated in economic literature that major economic variables display nonlinear behavior over the different phases of economic cycles. In this respect, complicated economic and finance systems are approached from a novel way.

Towards this direction, a novel nonlinear finance system with dissaving was studied, using tools from the nonlinear theory, such as bifurcation diagrams, Lyapunov exponents and phase portraits. During the simulation process, interesting phenomena concerning chaos theory, such as route to chaos through the mechanism of period doubling and crisis phenomena were observed.

Furthermore, from the simulation it was concluded that the inappropriate combination of the system's parameters was the cause of the undesirable chaotic behavior. When the interest rate $x$ is adjusted properly, the dissaving amount $a$ is kept at proper levels. If the above condition is met, prices are stabilized and society can be kept balanced. Also, the study results showed that the smaller the dissaving amount $a$ was, the greater the fluctuation of the system was. In more detail, when $a$ was very small, it caused chaotic behavior. However, the dissaving amount $a$ could not be too large because it caused system to lack vigour.

Furthermore, as the value of $c$ was smaller than one, the system remained always in periodic state, while as the elasticity of demand of commercial markets $c$ increased the regions of chaotic behavior were enlarged. So, this parameter can be kept into small values so as to avoid the undesirable system's chaotic behavior.

As next conclusion, one could say that for small values of $b$ the finance system's behavior alternated between chaotic and periodic one, as the parameter $c$ increased. However, as the value of the cost per-investment ratio $b$ increased the regions of system's periodic behavior were enlarged. Therefore, for large values of $b$ the system's chaotic behavior can be avoided.

It is interesting to be noted, that for the quick and healthy development of an economy, which presents chaotic behavior, it is crucial for its indexes to be forecasted and controlled. This was the beginning of the study of an interesting scheme of adaptive control of the proposed finance system's behavior.

Finally, the novel nonlinear finance system was studied from the engineering point of view by emulating this system with an electronic circuit. The simulation results of circuit's behavior were studied by using the package Cadence OrCAD. The results confirmed the feasibility of the theoretical model. 


\section{References}

[1] G. CHEN and X. DONG: From chaos to order: Methodologies, perspectives and applications. Singapore, World Scientific, 1998.

[2] I.R. EPSTEIN and J.A. POJMAN: An introduction to nonlinear chemical dynamics: Oscillations, waves, patterns, and chaos. (Topics in Physical Chemistry) Oxford, Oxford University Press, 1998.

[3] J. WALLECZEK: Self-organized biological dynamics and nonlinear control: Toward understanding complexity, chaos and emergent function in living systems. Cambridge, Cambridge University Press, 2006.

[4] S.H. STROGATZ: Nonlinear dynamics and chaos: With applications to physics, biology, chemistry, and engineering. USA, Westview Press, 2001.

[5] L. PRIBYLOVA: Bifurcation routes to chaos in an extended van der Pol's equation applied to economic models. Electr. J. Differ. Equ., 2009 (2009), 1-21.

[6] A.L. CHIAN, F.A. BOROTTO, E.L. REMPEL and C. ROGERS: Attractor merging crisis in chaotic business cycles. Chaos Soliton Fract., 24 (2005), 869-875.

[7] L.D. CESARE and M. SPORTELLI: A dynamic IS-LM model with delayed taxation revenues. Chaos Soliton Fract., 25 (2005), 233-244.

[8] H.W. LORENZ and H.E. NUSSE: Chaotic attractors, chaotic saddles, and fractal basin boundaries: Goodwin's nonlinear accelerator model reconsidered. Chaos Soliton Fract., 13 (2002), 957-965.

[9] J.H. MA and Y.S. CHEN: Study for the bifurcation topological structure and the global complicated character of a kind of nonlinear finance system (I). Appl. Math. Mech. (English ed.), 22 (2001), 1240-1251.

[10] J.H. MA and Y.S. CHEN: Study for the bifurcation topological structure and the global complicated character of a kind of nonlinear finance system (II). Appl. Math. Mech. (English ed.), 22 (2001), 1375-1382.

[11] W.C. CHEN: Nonlinear dynamics and chaos in a fractional-order financial system. Chaos Soliton Fract., 36 (2008), 1305-1314.

[12] Ch.K. VOLOS, I.M. KYPRIANIDIS, S.G. STAVRINIDES, I.N. STOUBOULOS, I. MAGAFAS and A.N. ANAGNOSTOPOULOS: Nonlinear financial dynamics from an engineer's point of view. J. of Engineering Science and Technology Review, 4 (2011), 281-285.

[13] Ch.K. VOLOS, I.M. KYPRIANIDIS and I.N. STOUBOULOS: Synchronization phenomena in coupled nonlinear systems applied in economic cycles. WSEAS Trans. Syst., 11 (2012), 681-690. 
[14] Ch.K. VOLOS, I.M. KYPRINAIDIS and I.N. STOUBOULOS: The effect of foreign direct investment in economic growth from the perspective of nonlinear dynamics. J. of Engineering Science and Technology Review, 8 (2015), 1-7.

[15] J.H. POINCARE: Sur le probléme des trois corps et les équations de la dynamique, Divergence des series de M. Lindstedt. Acta Mathematica, 13 (1890), $1-270$.

[16] E.N. LORENZ: Deterministic non-periodic flow. J. Atm. Sciences, 20 (1963), 130-141.

[17] B. MANDELBROT: The fractal geometry of nature. New York, W.H. Freeman and Company, 1977.

[18] P.S. LAPLACE: Traité du mécanique céleste, oeuvres complètes de Laplace. Paris, Gauthier-Villars, 1825.

[19] R.M. MAY and A.R. MCLEAN: Theoretical ecology: principles and applications. Oxford, Blackwell, 2007.

[20] C. KYRTSOU and C. VORLOW: Complex dynamics in macroeconomics: A novel approach. New Trends in Macroeconomics, C. Diebolt, and C. Kyrtsou, Berlin, Springer Verlag, (2005), 223-238.

[21] B. VAN DER POL and J. VAN DER MARK: Frequency demultiplication. Nature, 120 (1927), 363-364.

[22] L.W. CASPERSON: Gas laser instabilities and their interpretation. In: Proc. of the NATO Advanced Study Institute, Springer Verlag, (1988), 83-98.

[23] R.J. FIELD and L. GYORGYI: Chaos in chemistry and biochemistry. Singapore, World Scientific Publishing, 1993.

[24] G.L. BAKER: Chaotic dynamics: An introduction. Cambridge, Cambridge University Press, 1996.

[25] F.C. MOON: Chaotic vibrations: An introduction for applied scientists and engineers. USA, Wiley, 1987.

[26] Q. GAO and J. MA: Chaos and Hopf bifurcation of a finance system. Nonlinear Dynam., 58 (2009), 209-216.

[27] A. WOLF, J.B. SWIFT, H.L. SWINNEY and J.A. VASTANO: Determining Lyapunov exponents from a time series. Physica D, 16 (1985), 285-317.

[28] C. JEFFRIES and J. PEREZ: Direct observation of crises of the chaotic attractor in a nonlinear oscillator. Phys. Rev. A, 27 (1983), 601-603. 
[29] H.K. KHALIL: Nonlinear systems. (3rd edn.) USA, Prentice Hall, New Jersey, 2001.

[30] M. BARAKAT, A. MANSINGKA, A.G. RADWAN and K.N. SALAMA: Generalized hardware post processing technique for chaos-based pseudorandom number generators. ETRI J., 35 (2013), 448-458.

[31] L. GAMEZ-GUZMAN, C. CRUZ-HERNADEZ, R. LOPEZ-GUTIERREZ, E.E. GARCIA-GUERRERO: Synchronization of Chua's circuits with multiscroll attractors: Application to communication. Commun. Nonlinear Sci. Numer. Simul., 14 (2009), 2765-2775.

[32] S. SADOUDI, C. TANOUGAST, M.S. AZZAZ and A. DANDACHE: Design and FPGA implementation of a wireless hyperchaotic communication system for secure realtime image transmission. EURASIP J. Image and Video Processing, 943 (2013), 1-18.

[33] Ch. K. VOLOS, I. M. KYPRIANIDIS and I. N. STOUBOULOS: A chaotic path planning generator for autonomous mobile robots. Robot. Auto. Systems, 60 (2012), 651-656.

[34] Ch.K. VOLOS, I.M. KYPRIANIDIS and I.N. STOUBOULOS: Image encryption process based on chaotic synchronization phenomena. Signal Process., 93 (2013), $328-1340$.

[35] M.E. YALCIN, J.A.K. SUYKENS and J. VANDEWALLE: True random bit generation from a double-scroll attractor. IEEE Trans. Circuits Syst. I, Reg. Papers, 51 (2004), 1395-1404.

[36] A. BUSCARINO, L. FORTUNA and M. FRASCA: Experimental robust synchronization of hyperchaotic circuits. Physica D, 238 (2009), 1917-1922.

[37] V. SUNDARAPANDIAN and I. PEHLIVAN: Analysis, control, synchronization, and circuit design of a novel chaotic system. Math. Comp. Modelling, 55 (2012), 1904-1915.

[38] S. BOUALI, A. BUSCARINO, L. FORTUNA, M. FRASCA and L.V. GAMBUZZA: Emulating complex business cycles by using an electronic analogue. Nonl. Anal.: Real World Applications, 13 (2012), 2459-2465.

[39] L. FORTUNA, M. FRASCA and M.G. XIBILIA: Chua's circuit implementation: Yesterday, today and tomorrow. Singapore, World Scientific, 2009. 